\title{
Fractals, Log-Periodicity and Financial Crashes
}

\author{
P. OŚwięcimka ${ }^{a}$, S. Drożd $\dot{Z}^{a, b}$, J. Kwapień ${ }^{a}$, And A.Z. Górski ${ }^{a}$ \\ ${ }^{a}$ Institute of Nuclear Physics, Polish Academy of Sciences, E. Radzikowskiego 152, PL-31-342 Kraków, Poland \\ ${ }^{b}$ Faculty of Mathematics and Natural Sciences, University of Rzeszów, PL-35-310, Rzeszów, Poland \\ Presence of self-similar patterns in the financial dynamics is by now well established and even convincingly \\ quantified within the multifractal formalism. Here we focus attention on one particular aspect of this self-similarity \\ which potentially is related to the discrete-scale invariance underlying the system composition and manifests itself \\ by the log-periodic oscillations cascading self-similarly through various time scales. Such oscillations accumulate \\ at the turning (critical) points that in the financial dynamics are often identified as crashes. This property thus \\ allows us to develop a methodology that may be useful also for prediction. A model Weierstrass-type function is \\ used to illustrate the relevant effects and several examples demonstrating that such effects in the real financial \\ markets take place indeed, are reviewed.
}

PACS numbers: 52.35.Mw, 05.45.Df, 05.70.Jk

\section{Introduction}

Fractality is a concept that pervades all the science. The famous question "How long is the coast of Britain" [1] becomes well-founded and an answer quantifiable within the fractal formalism of fractal dimensions. Many other static objects as well as the dynamical processes that Nature is abundant with fall into the same category [2]. The same applies to the parameters representing dynamics of the financial assets. The length of the graph representing the price changes increases with increasing the time resolution with no signal for its convergence to any finite number and - for a sufficiently liquid market — such a graph remains "rough" at any such scale. This property of the financial graphs can visually be well illustrated by the Weierstrass-type function $[3,4]$

$$
f(t)=\sum_{n=0}^{\infty} \frac{1}{\lambda^{(2-D) n}} \exp \left(-\lambda^{n} t \cos (\gamma)\right) \cos \left(\lambda^{n} t \sin (\gamma)\right),
$$

where $\lambda$ and $\gamma \in[0, \pi / 2]$ are parameters that fix its structure. For $\gamma=\pi / 2$ the function develops a fractal structure and the parameter $D$ corresponds to the fractal dimension of this function. It then has the remarkable property of being continuous but nowhere differentiable. An example of such a function for $D=1.4$ and $\lambda=2$, with the summation truncated at $n=50$, is shown in Fig. 1. As the two successive steps of magnification clearly illustrate, this function exhibits the property of self-similarity.

\section{Fractal log-periodicity}

It is even more interesting to see that this self-similarity involves a very special pattern. The intervals between the subsequent most pronounced peaks at one particular $i$-th level of magnification get contracted by

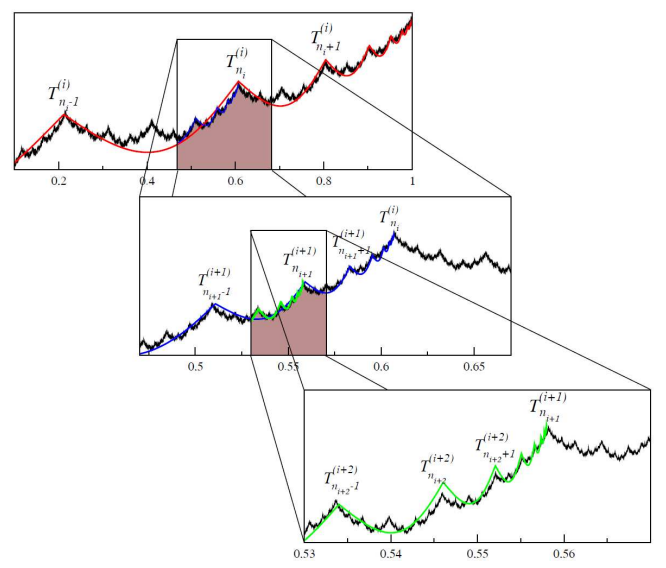

Fig. 1. The Weierstrass-type function of Eq. (1) for $\gamma=\pi / 2, D=1.4$ and $\lambda=2$. The sum in Eq. (1) has been truncated at $N=50$ because the function does not change significantly beyond this value of $N$. Two consecutive levels of magnification are also shown in order to demonstrate its self-similar character. The symbols $T_{n_{i}}^{(i)}$ mark the maksima at the $i$-th level of magnification.

the same constant factor $\lambda>1$ according to

$$
\frac{T_{n_{i}+1}^{(i)}-T_{n_{i}}^{(i)}}{T_{n_{i}}^{(i)}-T_{n_{i}-1}^{(i)}}=\frac{1}{\lambda} .
$$

These intervals thus form a geometric progression whose all terms can be summed up to infinity thus determining the accumulation point $T_{\mathrm{c}}$. In fact, any pronounced peak at $T_{n_{i}}^{(i)}$ for a fixed $i$-th level of magnification can be considered such an accumulation point $T_{\mathrm{c}}$ as it is proceeded by an infinite sequence of analogous peaks $T_{n_{i+1}}^{(i+1)}$ that constitute its counterparts at the deeper $(i+1)$-th level of magnification and fulfil the following relation: 


$$
T_{n_{i}}^{(i)}=\frac{T_{n_{(i+1)}}^{(i+1)} T_{n_{(i+1)}}^{(i+1)}-T_{n_{(i+1)}+1}^{(i+1)} T_{n_{(i+1)}-1}^{(i+1)}}{2 T_{n_{(i+1)}}^{(i+1)}-T_{n_{(i+1)}+1}^{(i+1)}-T_{n_{(i+1)}-1}^{(i+1)}} .
$$

This relation thus sets a strict correspondence between the turning points - in the graph seen as cusps - and the locations of the three consecutive such points determine position of the next and thus, by iteration, the structure of the whole hierarchy at all scales.

In simple and smooth terms the essential elements in Fig. 1 indicated by the thin solid lines - of the basic pattern of this structure at any particular level of magnification can be represented by a function $\phi(T)$ of the type

$$
\begin{aligned}
& \phi(T)=A+B\left|T_{\mathrm{c}}-T\right|^{\alpha} \\
& \quad+C\left|T_{\mathrm{c}}-T\right|^{\alpha}\left|\cos \left(\omega \ln \left|T_{\mathrm{c}}-T\right|-\varphi\right)\right|,
\end{aligned}
$$

where $A, B$, and $C$ are constants, $\alpha<0$ and $\omega=$ $2 \pi / \ln (\lambda)$. The dominant power-law behaviour, a hallmark of all critical phenomena, and the log-periodic corrections to the leading term are the main features of this function.

The Weierstrass-type function turns out a particular solution of the discrete renormalization group equation for critical phenomena $[4,5]$. This fact - in view of the suggestion that the financial markets may be governed by phenomena analogous to criticality in the statistical physics sense [5] — as well as abundance of the cups-like shapes in the financial dynamics justifies searching for parallels. Indeed, as it is shown in Fig. 2 which presents the time evolution of the German stock index DAX over the period of approximately first ten months in 1998 versus the best fit in terms of Eq. (4), the main features are amazingly similar. Even more, it is remarkable in this example that imprints of the contraction of oscillations in the spirit of Eq. (2) and thus also of Eq. (3) in the real market may cascade self-similarly through several time scales [6], resembling the model Weierstrass-type function. The most relevant - as it fixes the ratio of the contraction of oscillations - parameter, which is $\lambda$, here equals 2 .

\section{Financial markets}

The real financial dynamics is typically much more involved than just the model Weierstrass function or even than the selected DAX case shown in Fig. 2. Nevertheless, even in a much more complex evolution of the financial patterns one almost always finds components that can be well represented by Eq. (4) at several levels of the time resolution. In Fig. 3 the S\&P500 development over a very interesting and rich in diversity of different market phases time period of years 2000-2009 versus its optimal interpretation in terms of the basic formula prescribed by Eq. (4) is illustrated. Indeed, both variants, accelerating $T<T_{\mathrm{c}}$ as well as decelerating $T>T_{\mathrm{c}}$ of this formula can be here recognized to properly reflect

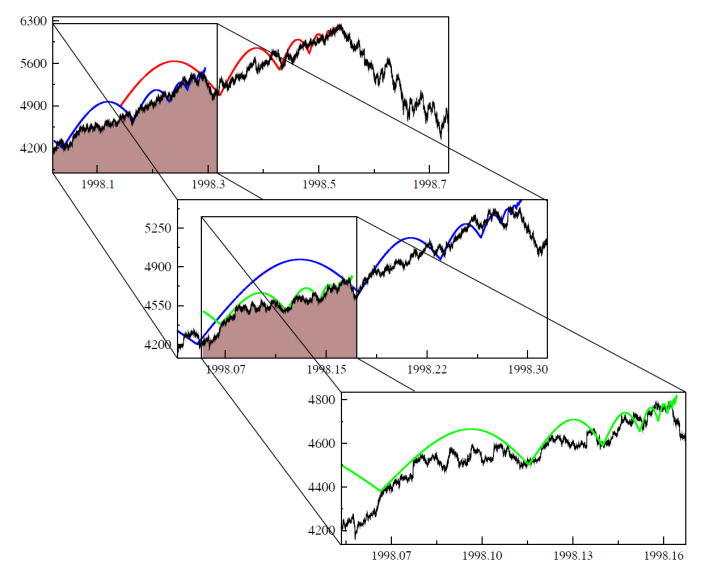

Fig. 2. Time evolution of the German DAX index over the period of approximately first ten months in 1998 together with the best logperiodic fit in three levels of magnification.

the oscillation structure and the accumulation of oscillations always coincides with the trend reversal. Furthermore, irrespective of the time scale at which it operates, the optimal value of the contraction parameter is $\lambda=2$, both in the bull and in the bear market phase.

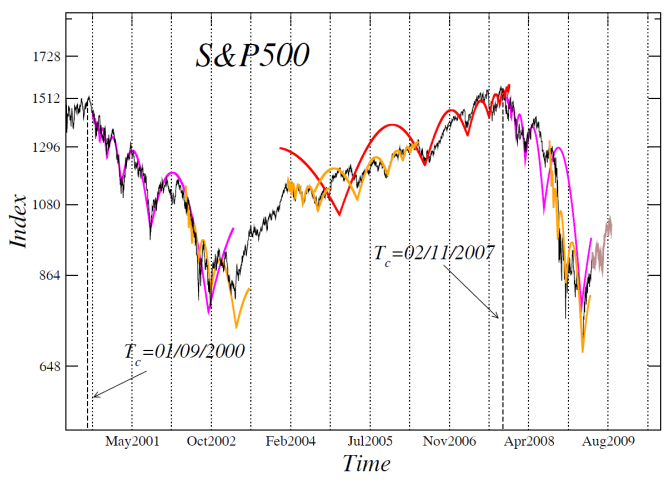

Fig. 3. The 2000 — present history of the S\&P500 index interpreted in terms of the optimal log-periodic representations for different phases of its development.

A particularly appealing aspect of the patterns described above is that they carry a potential for prediction. Suppose that during an increase a three consecutive repetitive structures, either minima or maxima, labeled as $T_{n_{i}}^{(i)}, T_{n_{i}+1}^{(i)}$, and $T_{n_{i}+2}^{(i)}$, respectively, are identified on a particular $i$-th level of the time resolution. Then applying procedure analogous to Eq. (3), or its equivalent expressed by Eq. (4), the corresponding $T_{\mathrm{c}}$ which marks termination of this increasing phase can be evaluated in advance. In view of an unavoidable frequent imprecision in identifying such repetitive structures in the real market dynamics the further constraints are put on such a procedure the more reliable it can be made. One crucial related element is an original postulate [6] that for the stock markets the contraction factor $\lambda \approx 2$ and is 
the same for all the time scales. Later on this postulate was further verified and confirmed $[7,8]$ for several world leading stock markets. More recent studies based on the precious metal market [9] and on the oil market [10] allow one to extend this universality aspect of the parameter $\lambda$ also to the commodity market. These last two cases were in addition associated with the real predictions as documented in the corresponding references. This fact of course even strengthens the above indication concerning such a specific value of the parameter $\lambda$.

Especially spectacular was the precision of prediction [10] of the oil price reversal that occurred on July 11, 2008, exactly as predicted [10]. Figure 4 shows the oil price development over the time period June 2007December 2008 and thus extends somewhat longer than the corresponding Fig. 2 of Ref. [10]. This extension even better documents a highly speculative character of the oil price dynamics. Since June 11, 2008 until the end of the same year the oil price dropped down by a factor of three and this drawdown was accompanied by the decelerating log-periodic oscillations and, as also indicated in Fig. 4, even a smaller time-scale substructure in the end of this declining phase emerged. Of course, the theoretical representation in terms of Eq. (4) corresponds to $\lambda=2$ on both sides of the transition point which provides another argument in favor of the universality of this parameter value.

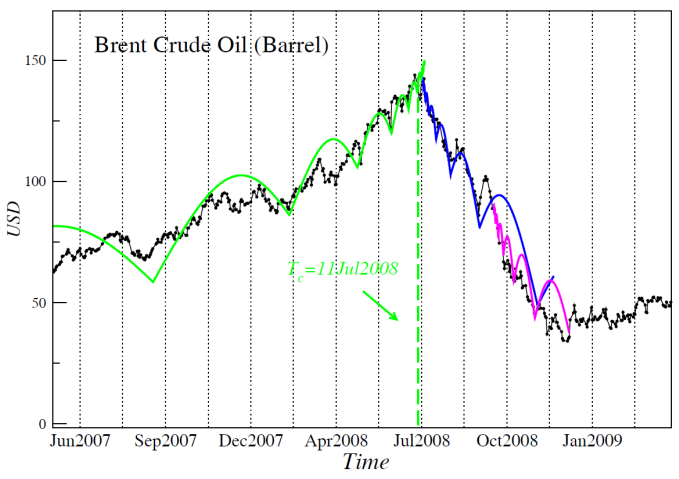

Fig. 4. Temporal evolution of the brent crude oil price over the period June 2007-December 2008 with the best-fitted logperiodic functions modeling the bull and the bear phases.

From the longer time-perspective the current oil price dynamics can be viewed as it is shown in Fig. 5. This way of interpretation was postulated already in Ref. [10] and is based on a phenomenon that is termed a "superbubble" [7]. Accordingly, the oil bubble that did burst on July 11, 2008 was a bubble built on top (therefore a "superbubble") of a longer term bubble that is still in action. The fact that after the decline the oil price returned to the level consistent with the long term trend indicates that such an interpretation makes a lot of sense. A systematic elevation of the oil price, decorated by the log-periodic oscillations within the tunnel as sketched in Fig. 5, is thus likely to last until almost the end of 2010.

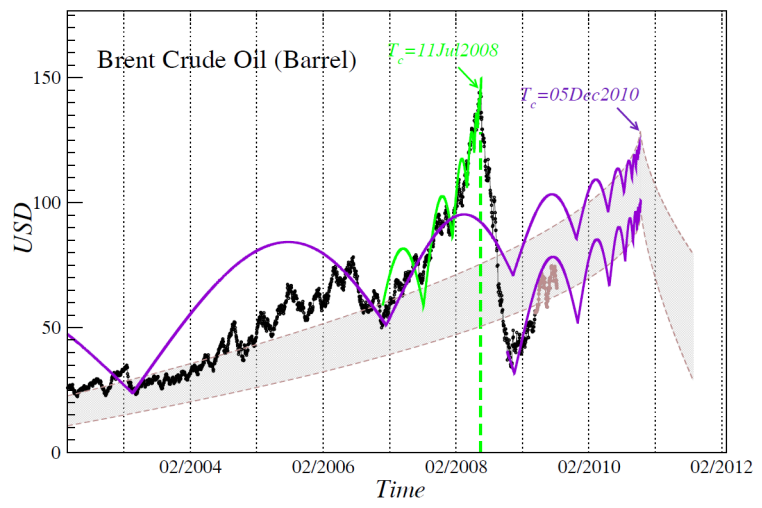

Fig. 5. Long-term temporal evolution of the brent crude oil price over the years 2002-2009 and the possible long-term logperiodic trends culminating on 5 Dec 2010 .

\section{Conclusions}

In our paper we have shown that the evolution of stock indices can be described by a self similar structure of log-periodic oscillations on different levels of temporal resolution. This cascade of log-periodic patterns resembles the structure of the Weierstrass function, in which the oscillations accelerate forming an infinite sequence of cusps that converge to a local accumulation point, which in turn is one of the serial cusps in the higher-level convergent sequence. As the presented example of the oil price shows, the identification of an accelerating or decelerating oscillating patterns in the data has also a strong forecasting potential. We have also stressed the possibility that the contracting factor $\lambda \approx 2$ can be universal.

\section{References}

[1] B.B. Mandelbrot, Science 155, 638 (1967).

[2] B.B. Mandelbrot, The fractal geometry of Nature, W.H. Freeman, San Francisco 1982.

[3] M.V. Berry, Z.V. Lewis, Proc. R. Soc. Lond. A 370, 459 (1980).

[4] S. Gluzman, D. Sornette, Phys. Rev. E 65, 036142 (2002).

[5] D. Sornette, Why Stock Markets Crash: Critical Events in Complex Financial Systems, Princeton University Press, Princeton 2003.

[6] S. Drożdż, F. Ruf, J. Speth, M. Wójcik, Eur. Phys. J. B 10, 589 (1999).

[7] S. Drożdż, F. Grümmer, F. Ruf, J. Speth, Physica A 324, 174 (2003).

[8] M. Bartolozzi, S. Drożdż, D.B. Leinweber, J. Speth, A.W. Thomas, Int. J. Mod. Phys. C 16, 1347 (2005).

[9] S. Drożdż, J. Kwapień, P. Oświęcimka, J. Speth, Acta Phys. Pol. A 114, 539 (2008).

[10] S. Drożdż, J. Kwapień, P. Oświęcimka, Acta Phys. Pol. A 114, 699 (2008). 